\title{
Inflammatory Ly6Chi monocytes and their conversion to M2 macrophages drive atherosclerosis regression
}

\author{
Karishma Rahman, ${ }^{1}$ Yuliya Vengrenyuk, ${ }^{2}$ Stephen A. Ramsey, ${ }^{3}$ Noemi Rotllan Vila, ${ }^{4}$ Natasha M. Girgis, ${ }^{5}$ Jianhua Liu, ${ }^{6}$ \\ Viktoria Gusarova, ${ }^{7}$ Jesper Gromada, ${ }^{7}$ Ada Weinstock, ${ }^{1}$ Kathryn J. Moore, ${ }^{1}$ P'ng Loke, ${ }^{8}$ and Edward A. Fisher ${ }^{1}$ \\ 'Departments of Medicine (Cardiology) and Cell Biology, and the Marc and Ruti Bell Program in Vascular Biology, New York University School of Medicine, New York, New York, USA. ²Department \\ of Cardiology, Mount Sinai School of Medicine, New York, New York, USA. ${ }^{3}$ Department of Biomedical Sciences and School of Electrical Engineering and Computer Science, Oregon State University, \\ Corvallis, Oregon, USA. ${ }^{4}$ Department of Vascular Biology and Therapeutics Program, and Integrative Cell Signaling and Neurobiology of Metabolism Program, Section of Comparative Medicine and \\ Department of Pathology, Yale University School of Medicine, New Haven, Connecticut, USA. ${ }^{5}$ Constellation Pharmaceuticals, Cambridge, Massachusetts, USA. ${ }^{6}$ Department of Surgery, Mount Sinai \\ School of Medicine, New York, New York, USA. ${ }^{7}$ Regeneron Pharmaceuticals, Tarrytown, New York, USA. ${ }^{8}$ Department of Microbiology, New York University School of Medicine, New York, New York, USA.
}

\begin{abstract}
Atherosclerosis is a chronic inflammatory disease, and developing therapies to promote its regression is an important clinical goal. We previously established that atherosclerosis regression is characterized by an overall decrease in plaque macrophages and enrichment in markers of alternatively activated M2 macrophages. We have now investigated the origin and functional requirement for $\mathrm{M} 2$ macrophages in regression in normolipidemic mice that received transplants of atherosclerotic aortic segments. We compared plaque regression in WT normolipidemic recipients and those deficient in chemokine receptors necessary to recruit inflammatory Ly6 $\mathrm{C}^{\mathrm{hi}}\left(\mathrm{Ccr2}^{-/-}\right.$or $\left.\mathrm{Cx} 3 \mathrm{cr}^{-/-}\right)$or patrolling Ly6 $\mathrm{C}^{10}\left(\mathrm{Ccr5}^{-/-}\right)$monocytes. Atherosclerotic plaques transplanted into WT or $\mathrm{Ccr}^{-/-}$recipients showed reduced macrophage content and increased M2 markers consistent with plaque regression, whereas plaques transplanted into $\mathrm{Crr}^{-/-}$or $\mathrm{Cx}_{3} \mathrm{cr}^{-/-}$recipients lacked this regression signature. The requirement of recipient $L y 6 C^{h i}$ monocyte recruitment was confirmed in cell trafficking studies. Fate-mapping and single-cell RNA sequencing studies also showed that M2-like macrophages were derived from newly recruited monocytes. Furthermore, we used recipient mice deficient in STAT6 to demonstrate a requirement for this critical component of M2 polarization in atherosclerosis regression. Collectively, these results suggest that continued

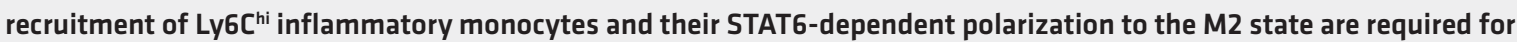
resolution of atherosclerotic inflammation and plaque regression.
\end{abstract}

\section{Introduction}

Despite major advances in treatment, atherosclerosis through its clinical sequelae, heart attack and stroke, is the leading cause of death worldwide. Regression of atherosclerosis is a vital goal in the quest to reduce cardiovascular risk, given that virtually all people by their fourth decade of life have established arterial plaques, some of which may ultimately become the culprit lesions causing clinical events.

To better understand the process of atherosclerosis regression, we and our collaborators have developed a number of surgical and non-surgical mouse models (1-4). In the surgical model, an APOE-deficient (Apoe $\left.e^{-/}\right)$C57BL/6 mouse aortic arch segment with atherosclerotic plaques is transplanted into the abdominal aorta of a WT C57BL/6 recipient mouse. This sudden and sustained normalization of dyslipidemia leads to remarkably rapid decreases in both plaque size and the content of cells positive for

Authorship note: K. Rahman and Y. Vengrenyuk contributed equally to this work. P. Loke and E.A. Fisher contributed equally to this work.

Conflict of interest: V. Gusarova and J. Gromada are employees and shareholders of Regeneron Pharmaceuticals. N.M. Girgis has been an employee of Janssen Research and Development and is currently an employee of Constellation Pharmaceuticals.

Submitted: January 2, 2014; Accepted: May 4, 2017.

Reference information: / Clin Invest. 2017;127(8):2904-2915.

https://doi.org/10.1172/JCI75005. the standard macrophage marker CD68 (5-8). Reductions in the same parameters have also been noted in other mouse models of atherosclerosis regression in which normalization of dyslipidemia was induced by non-surgical means (reviewed in ref. 9).

One influential classification of macrophages is based on the work of Siamon Gordon and Alberto Mantovani and their colleagues, who have broadly described M1 (classically activated) and M2 (alternatively activated) states $(10,11)$. In vitro, macrophages can polarize toward either the M1 or M2 state by treatment with IFN- $\gamma$ and LPS, or IL-4 and IL-13, respectively. Notably, in regressing atherosclerotic plaques, we have observed that there is reduced expression of classical inflammatory genes characteristic of M1 macrophages, such as monocyte chemoattractant protein-1 (MCP-1), TNF- $\alpha$, and iNOS, coincident with increased expression of genes encoding markers of alternatively activated, tissueremodeling M2 macrophages, such as arginase 1 (ARG1), mannose receptor (MR, also known as CD206), and IL-10 in CD68 ${ }^{+}$cells $(2,3,5,12,13)$. Importantly, the enrichment of plaque $\mathrm{CD}^{2} 8^{+}$cells with markers of the M2 phenotype, though initially discovered in the transplant model $(5,12)$, has been subsequently found in several different models of atherosclerosis regression (2-4), suggesting this represents a signature of plaque regression.

The mechanisms by which the macrophage phenotype changes from M1 to M2 in regressing plaques and whether this shift is 
required for regression remain unknown. Based on one study of macrophage phenotypes during the progression of atherosclerosis in $A_{p o e^{-/}}$mice - in which serial immunohistological examinations showed that plaque macrophages have M2 phenotypes at the early stages of the disease but become M1 macrophages as the lesions advance (14) - it is possible that M1 macrophages in the plaques retain plasticity and reclaim characteristics of the original M2 phenotype in the regression environment. Alternatively, the M2 macrophages may be derived through the proliferation of a pool of tissue-resident macrophages (15) that originated from the yolk sac, as has previously been demonstrated in other tissues (16). Finally, M2 macrophages in regressing plaques may be derived from newly recruited monocytes.

Based on our earlier studies showing continued recruitment of circulating monocytes into regressing plaques (17), we prioritized testing this third hypothesis. We focused on two key questions: (a) If newly recruited monocytes became M2 macrophages, which circulating monocyte subset provided the new cells? (b) Is the conversion to the M2 phenotype required for plaque regression?

There are two major subsets of murine monocytes, and they are traditionally defined by the expression of different chemokine receptors: $\mathrm{CCR} 2^{+} \mathrm{CX} 3 \mathrm{CR} 1^{+} \mathrm{Ly} 6 \mathrm{C}^{\text {hi }}$ and $\mathrm{CCR} 2{ }^{-} \mathrm{CX} 3 \mathrm{CR} 1^{++} \mathrm{Ly} 6 \mathrm{C}^{\mathrm{lo}}$ monocytes. These subsets are characterized by distinct migratory and inflammatory properties (reviewed in ref. 16). The classical Ly $6 \mathrm{C}^{\text {hi }}$ monocytes efficiently infiltrate inflammatory sites, dominating the acute inflammatory response to pathogens, as well as the monocytosis and recruitment into progressing plaques in Apoe ${ }^{-/}$mice $(18,19)$. They employ both CCR2 and CX3CR1 to enter atherosclerotic lesions and are thought to become M1 macrophages in most inflammatory sites (19-21). Thus, Ly6C ${ }^{\text {hi }}$ cells are often termed "inflammatory monocytes." In contrast, the nonclassical monocytes (CCR2 ${ }^{-} \mathrm{CX} 3 \mathrm{CR}^{++}{ }^{+} \mathrm{Ly} 6 \mathrm{C}^{\mathrm{lo}}$ ) do not express CCR2, but do express the highest levels of CX3CR1 (21). These monocytes patrol blood vessels and also accumulate at inflammatory sites, where they are thought to give rise to M2 macrophages (21). Because the Ly6 $\mathrm{C}^{\text {lo }}$ monocytes do not express CCR2, other chemokine receptors must be responsible for their recruitment to atherosclerotic plaques. Surprisingly, despite their high expression of CX3CR1, this chemokine receptor was dispensable for nonclassical monocyte recruitment, whereas CCR5 was not (19). Notably, the combined inhibition of CCR2, CX3CR1, and CCR5 essentially abolished atherosclerosis progression in hyperlipidemic mice, suggesting that recruitment of both subsets of circulating monocytes into plaques is required for their development (22).

To investigate the source of, and functional requirement for, M2 macrophages in atherosclerosis regression, we employed the aortic transplantation model and used recipient mice lacking the major receptors required for $\mathrm{Ly} 6 \mathrm{C}^{\text {hi }}$ and $\mathrm{Ly} 6 \mathrm{C}^{\mathrm{lo}}$ monocyte recruitment into developing plaques (e.g., CCR2 and CCR5, respectively). We show herein that recruitment of recipient CCR2-dependent Ly6 $\mathrm{C}^{\text {hi }}$ monocytes is essential for atherosclerosis regression and the concurrent reduction in plaque macrophage content and enrichment in M2 macrophages. Notably, enrichment in the M2 phenotype and reduction in macrophage content were both markedly attenuated in regressing plaques when the STAT6 signaling pathway, known to be downstream from the M2-polarizing cytokines IL-4/IL-13 (23), was absent in recipient mouse circulating monocytes. Over- all, these results indicate a previously unappreciated requirement for the recruitment of inflammatory Ly6 $\mathrm{C}^{\text {hi }}$ monocytes and their STAT6-dependent conversion to tissue-remodeling M2 macrophages in the regression of atherosclerosis.

\section{Results}

Deficiency of CCR2 or CX3CR1, but not CCR5, in aortic transplant recipients prevents plaque regression despite the reversal of dyslipidemia. At 4 weeks of age, Apoe $\mathrm{e}^{- \text {- }}$ mice were fed a standard high-fat high cholesterol diet ("Western diet" [WD]) to exacerbate their hyperlipidemia so that they developed advanced atherosclerotic lesions by 16 weeks (baseline group). The aortic arches from these donor mice were then transplanted into $\mathrm{Apoe}^{-/-}$, WT, $\mathrm{Crr} 2^{-/}, \mathrm{C} \times 3 \mathrm{crl}^{-/-}$, or $\mathrm{Ccr}^{-/-}$mice maintained on a chow diet, as illustrated in the experimental design in Figure 1A. In contrast to the hyperlipidemia of the Apoe $^{-/-}$recipients, which led to atherosclerosis progression, the lipoprotein profiles showed that the WT and chemokine receptor-deficient mice were all normolipidemic, with total plasma cholesterol levels of $\sim 90 \mathrm{mg} / \mathrm{dl}$ (vs. $1,100 \mathrm{mg} / \mathrm{dl}$ in the $A_{p o e^{-/}}$donors on WD and $400 \mathrm{mg} / \mathrm{dl}$ in the Apoe $e^{-/-}$recipients on chow; data not shown).

As we previously reported $(5,6,12,24)$, plaque area as well as macrophage (CD68 ${ }^{+}$cells) and neutral lipid (i.e., staining positive for Oil Red O) content in aortic arches transplanted into the regression environment of the WT mice were significantly decreased to $<50 \%$ of baseline by 5 days (Figure 1, B-E). Similarly, plaque, $\mathrm{CD}^{2} 8^{+}$, and lipid areas decreased in the $\mathrm{Crr}^{-/-}$recipients to a level comparable to that observed in WT recipients (Figure 1, B-E), consistent with regression of atherosclerosis. However, transfer of aortic segments into $\mathrm{Ccr} 2^{-/-}$or $\mathrm{C} x 3 \mathrm{cr}^{-/-}$recipients did not lead to significantly decreased plaque area or $\mathrm{CD} 8^{+}$cell content compared with baseline, despite plasma lipoprotein levels similar to those in WT recipients (Figure 1, B, C, and E). Furthermore, CCR2 or CX3CR1 deficiency in transplant recipients prevented the decrease in plaque neutral lipid content observed in the WT and $\mathrm{Ccr} 5^{-/}$recipients (Figure 1, D and E). These results indicate that CCR2 and CX3CR1, which mediate the recruitment of Ly6 $\mathrm{C}^{\text {hi }}$ monocytes into progressing plaques $(19,25-27)$, are essential for the regression of atherosclerosis. Furthermore, although CCR5 contributes to atherosclerosis progression by recruiting Ly6 $\mathrm{C}^{\mathrm{lo}}$ monocytes (22), this chemokine receptor, and by extension recruitment of Ly $6 \mathrm{C}^{\mathrm{lo}}$ monocytes, is not required for atherosclerosis regression.

Deficiency of CCR2 or CX3CR1, but not CCR5, in transplant recipients prevents the accumulation of $M 2$ macrophages in regressing plaques. In various models of atherosclerosis regression, an enrichment of M2 macrophage markers accompanies the change in plaque content of $\mathrm{CD} 68^{+}$cells $(2,4,5,12)$. To determine whether the chemokine receptors CCR2, CX3CR1, and CCR5 contribute to the enrichment of M2 macrophages and the reduction of the M1 inflammatory phenotype in regressing plaques, we transplanted aortic segments into the recipient mouse groups as outlined in Figure 1A and examined the expression of the M1 macrophage marker MCP-1 and the M2 markers MR and ARG1 by immunohistochemical staining. As shown in Figure 2, A-C, the MR- and ARG1-positive areas were significantly increased, while MCP-1 staining was decreased, in plaques transferred to normolipidemic WT (as expected) and $\mathrm{Crr}^{-1-}$ recipient mice compared with those from $\mathrm{Apoe}^{-/-}$recipient mice. In contrast, the $\mathrm{MCP}-1^{+}, \mathrm{MR}^{+}$, and 
A

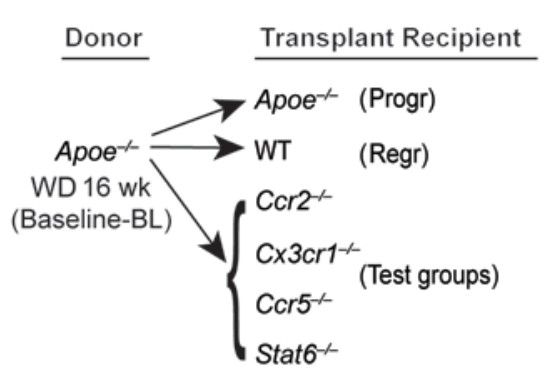

C

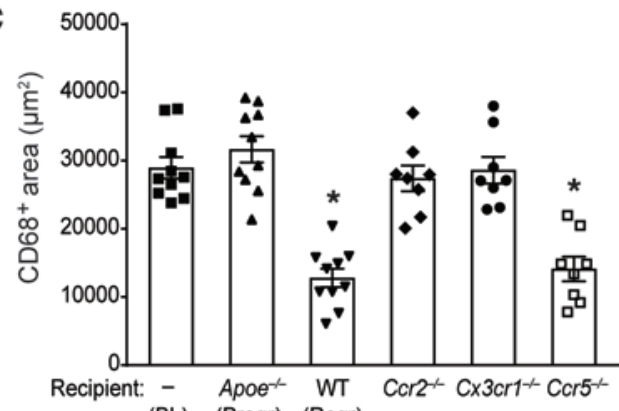

(BL) (Progr) (Regr)

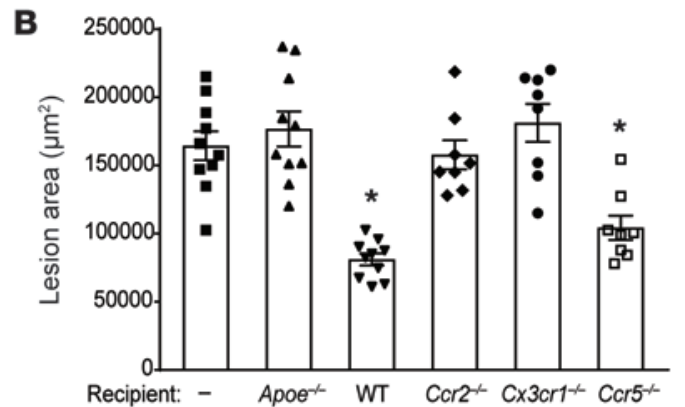

(BL) (Progr) (Regr)

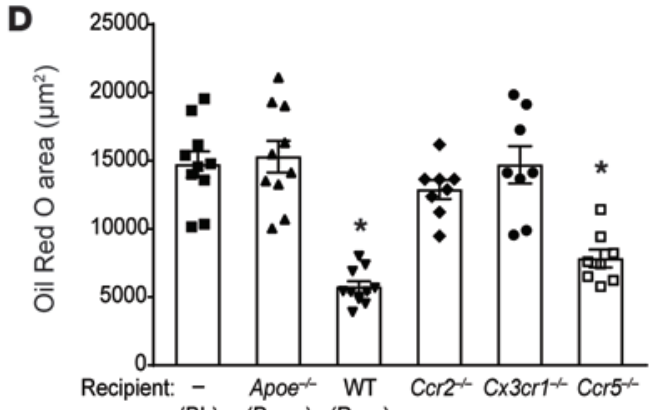

(BL) (Progr) (Regr)
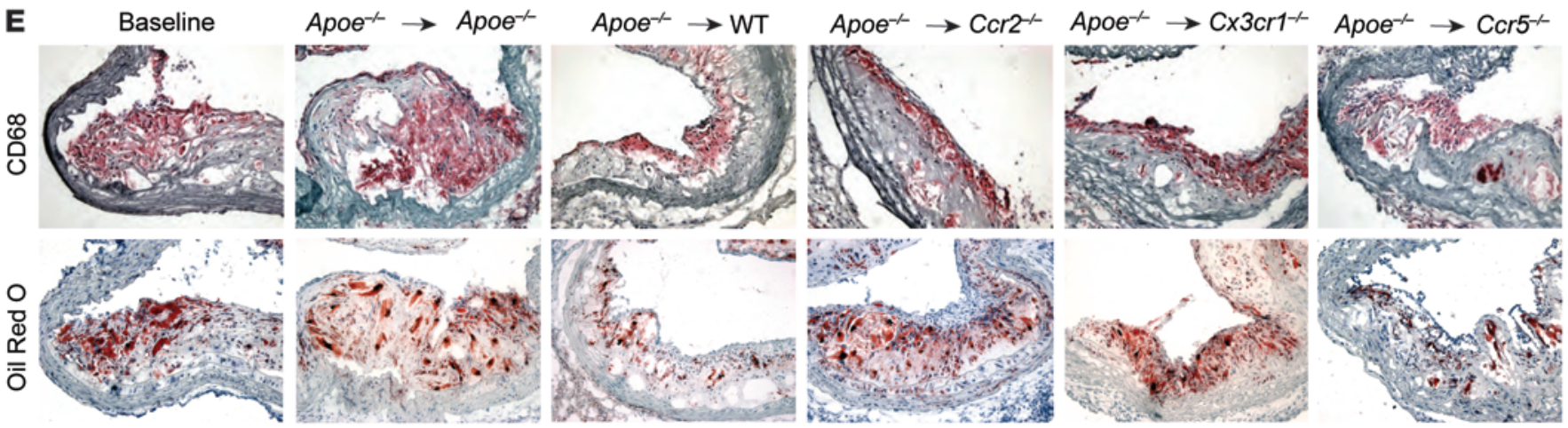

Figure 1. CCR2 and CX3CR1 are required for plaque regression. Analysis of aortic arch plaques from Apoe ${ }^{-/-}$mice on 16-week WD (baseline [BL]; $n=10$ ) or 5 days after transplantation into $A p o e^{-/-}$(progression [Progr]; $n=10$ ), WT (regression [Regr]; $n=10$ ), or chemokine receptor-KO recipient mice (Ccr2- ${ }^{-1-}$, $\mathrm{C} 3 \mathrm{Cr}^{-1-}$, or $\left.\mathrm{Cr} 5^{-1-} ; n=8\right)$. (A) Schematic of transplant experiments. Quantification of (B) lesion area, (C) immunohistochemical staining for the macrophage marker CD68, and (D) Oil Red 0 staining of neutral lipids. ${ }^{*} P<0.001$ compared with $\mathrm{BL}$ and Apoe ${ }^{-/-}$progression groups using 1-way ANOVA with Dunnett's multiple comparisons testing. (E) Representative sections of aortic transplant segments from BL and the recipient groups stained with antiCD68 antibody and Oil Red 0 , imaged at $\times 40$ magnification.

ARG1 ${ }^{+}$areas did not change significantly in plaques transplanted into $\mathrm{Ccr}^{--}$or $\mathrm{C} 3 \mathrm{crr}^{-/-}$recipient mice. Indeed, only a small proportion of plaque $\mathrm{CD}^{2} 8^{+}$cells in the $\mathrm{Ccr}^{-/}$and $\mathrm{C}_{3} 3 \mathrm{crr}^{-1-}$ recipients expressed MR or ARG1, while a significant majority of plaque $\mathrm{CD}^{+} 8^{+}$cells in WT and $\mathrm{Ccr}^{-}-$recipients were $\mathrm{MR}^{+}$and $\mathrm{ARG1}^{+}$(Figure 2, A-C). The results suggest that the recruitment of recipient monocytes through CCR2 and CX3CR1 is crucial not only for atherosclerosis regression, but also for the accumulation of M2 macrophages. These results, taken with the data in Figure 1, make the possibility that the conversion of M1 cells in the donor plaques is a significant source of M2 macrophages unlikely. Rather, they overwhelmingly appear to be derived from active recruitment of recipient monocytes from the Ly6C ${ }^{\text {hi }}$ subset. Although M2 macrophages have been proposed to arise from either the $\mathrm{Ly} 6 \mathrm{C}^{\text {lo }}$ subset (21) or from proliferation of tissue macrophages derived from the yolk sac
(16), our findings are in agreement with recent reports that Ly6 $\mathrm{C}^{\mathrm{hi}}$ inflammatory monocytes recruited to allergic skin, schistosome granulomas, or injured myocardium subsequently acquired the M2 phenotype (20, 28-31).

Acute inhibition of CCR2 prevents plaque regression and suppresses enrichment in the M2 macrophage phenotype. CCR2 is critical for both egress of Ly $6 \mathrm{C}^{\text {hi }}$ monocytes from bone marrow and for their entry into progressing atherosclerotic plaques; indeed, $\mathrm{Ccr}^{-} /$mice have reduced numbers of circulating monocytes and, when crossed to mouse models of atherosclerosis, have smaller plaques $(22,25,26)$. As the reduction of circulating monocytes in $\mathrm{Ccr}^{2--}$ mice may confound the interpretation of the aortic transplant experiments, we complemented this genetic approach with studies using a small molecule inhibitor of CCR2, which does not alter the total number of circulating monocytes or their subsets in 

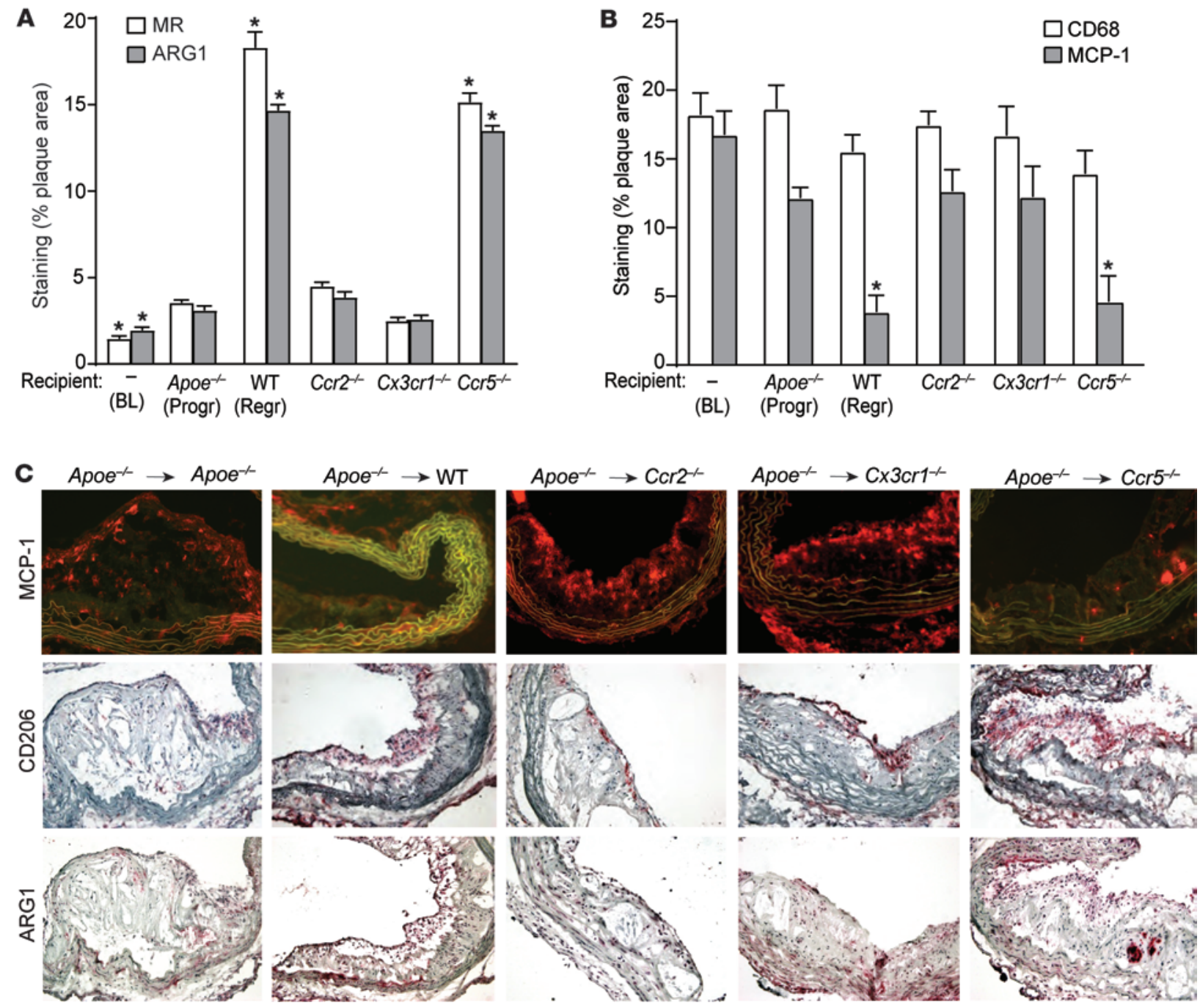

Figure 2. CCR2 and CX3CR1 are required for enrichment of M2 macrophages in the regressing plaque. Quantification of immunohistochemical staining for (A) markers of M2 macrophages MR and ARG1 and (B) the macrophage marker CD68 and an M1 macrophage marker MCP-1, in aortic arch plaques from Apoe $^{-/-}$mice on a 16-week WD (BL; $n=10$ ) or 5 days after transplantation into Apoe ${ }^{-/-}$(Progr; $\left.n=10\right)$, WT (Regr; $\left.n=10\right)$, or chemokine receptor-deficient recipient mice $\left(\mathrm{Crr}^{-1-}, \mathrm{Cx}_{3} \mathrm{Cr}^{-1-}\right.$ or $\left.\mathrm{CCr} 5^{-1-} ; n=8\right) .{ }^{*} P<0.01$ compared with $A p o e^{-/-}$progression group using 1-way ANOVA with Dunnett's multiple comparisons testing. (C) Representative images of aortic plaques stained for MCP-1, CD206 (MR), and ARG1, imaged at ×40 magnification.

short-term treatment (Figure 3, A and B). Notably, WT recipients treated with the CCR2 inhibitor for 48 hours before and 72 hours after the transplantation of aortic arches from $\mathrm{Apoe}^{-/-}$mice did not demonstrate lesion and $\mathrm{CD} 68^{+}$area reductions, in contrast to the WT recipients treated with vehicle (Figure $3, \mathrm{C}$ and $\mathrm{D}$ ). In addition, by immunostaining, CCR2 inhibition prevented the enrichment in the M2 marker MR and the decrease in the M1 marker MCP-1 that were observed in the WT-vehicle group (Figure 3E). These results not only are concordant with the consequences of the genetic deficiency of CCR2 (Figures 1 and 2), but also extend those data by showing that the role of CCR2 in atherosclerosis regression is independent of total circulating monocyte levels.

Assessment of monocyte trafficking shows Ly6C ${ }^{\text {hi }}$ monocyte recruitment is key to impaired regression in $\mathrm{Ccr}^{---}$mice. To extend the results with the inhibitor and to demonstrate that the impaired regression of plaques transplanted into $\mathrm{Ccr}^{-/}$recipient mice was specifically due to the reduced recruitment of the Ly $6 \mathrm{C}^{\text {hi }}$ subset of monocytes, we used two different labeling methods to track their recruitment into plaques. Using EdU (5-ethynyl-2'-deoxyuridine) to specifically label Ly6C $\mathrm{C}^{\text {hi }}(32-34)$ circulating monocytes (design shown in Figure 4A, labeling efficiency shown in Figure 4, B and C), we observed a significant reduction (relative to WT recipients) in EdU-labeled monocytes in plaques transplanted into $\mathrm{Ccr}_{2--}^{--}$ but not $\mathrm{Ccr}^{-/}$, mice (Figure 4D). This confirms that deficiency of CCR2 in recipient mice resulted in decreased recruitment of Ly $6 \mathrm{C}^{\mathrm{hi}}$ monocytes into plaques in a regression environment. Second, using fluorescent latex beads to specifically label circulating Ly $6 \mathrm{C}^{\text {lo }}$ $(8,17,19)$ monocytes (Figure $4, \mathrm{E}$ and $\mathrm{F})$, we observed that relative to WT recipients, there was a reduction $(P=0.05)$ in beadlabeled cells in plaques transplanted into $\mathrm{Ccr}^{--}$, but not $\mathrm{Ccr} 2^{--}$, recipient mice (Figure 4G). There were no significant differences in macrophage proliferation or apoptosis between the plaques transplanted into WT, $\mathrm{Ccr} 2^{-/}$, and $\mathrm{Ccr}^{-/}$recipients, as measured by staining for Ki67 (Figure 4H) and cleaved caspase 3 (Figure 4I), 

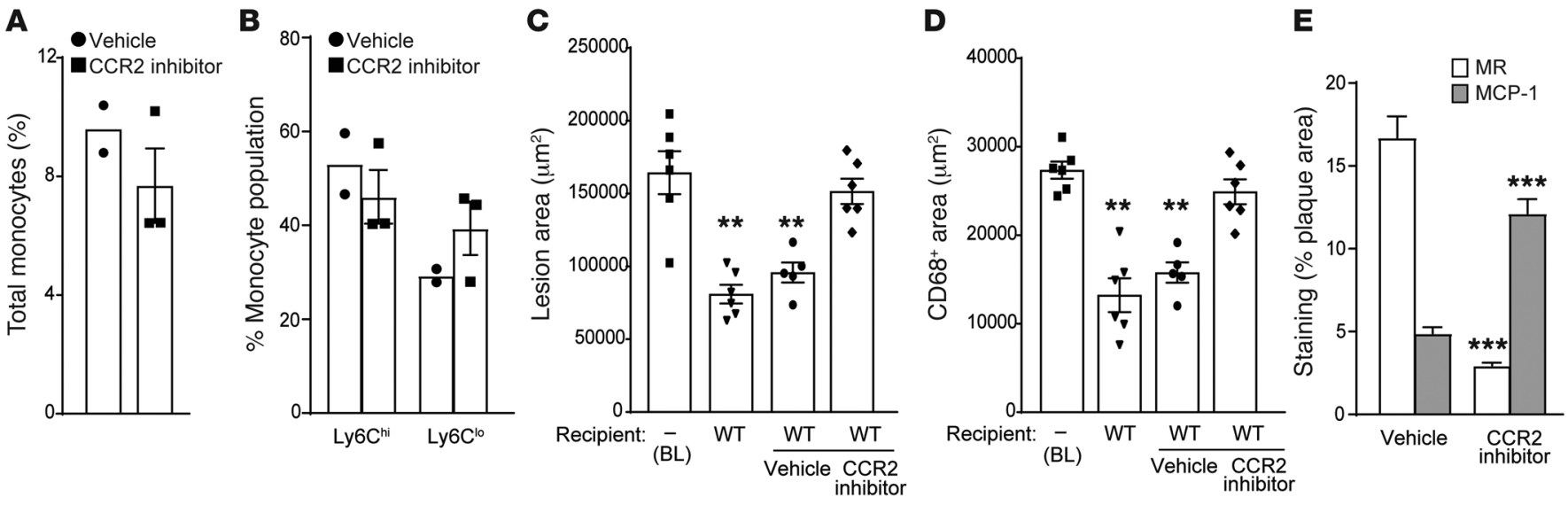

Figure 3. Effect of CCR2 inhibition on atherosclerosis regression and M2 macrophage enrichment. Effect of CCR2 inhibitor on the frequency of (A) total monocytes and (B) Ly6C ${ }^{\text {hi }}$ and Ly6C ${ }^{10}$ monocyte subsets in the blood of recipient mice (vehicle, $n=2$, and CCR2 inhibitor, $n=3$ ). (C-E) Quantification of (C) lesion area, (D) the macrophage marker CD68, and (E) MR and MCP-1 staining in aortic arch plaques from Apoe $e^{-/-}$mice on 16-week WD (BL; $\left.n=6\right)$ or 3 days after transplant into WT mice (regression environment) that were untreated $(n=6)$ or treated with vehicle $(n=5)$ or a CCR2 inhibitor $(n=6)$. For $\mathbf{C}$ and $\mathbf{D}$ : ${ }^{* *} P<0.001$ compared with BL using 1-way ANOVA with Dunnett's multiple comparisons testing. For $\mathbf{E},{ }^{* * *} P<0.0001$, CCR2 inhibitor group compared with vehicle group using unpaired $t$ testing.

respectively. As plaques transplanted into $\mathrm{Ccr}^{-/-}$recipient mice show regression similar to those in WT mice, despite the lack of recruitment $\mathrm{Ly} 6 \mathrm{C}^{\text {lo }}$ monocytes, this suggests that this monocyte population is not required for the promotion of atherosclerosis regression. Furthermore, the recruitment of Ly6 $6 \mathrm{C}^{\mathrm{lo}}$ monocytes into plaques is intact in $\mathrm{Ccr} 2^{-/-}$recipient mice, yet regression fails to occur, indicating that $\mathrm{Ly} 6 \mathrm{C}^{\mathrm{lo}}$ monocyte-derived cells cannot replace $\mathrm{Ly} 6 \mathrm{C}^{\mathrm{hi}}$ monocytes in promoting plaque regression.

M2 macrophages originate from newly recruited cells from recipient mice. Though there is concurrent recruitment of recipient monocytes and enrichment in plaque $\mathrm{CD}^{+} 8^{+}$cells with macrophage M2 characteristics, this does not definitely establish a direct link between these two findings. To demonstrate, using multiple approaches, that recipient mouse monocytes are indeed the source of M2 macrophages in regressing plaques, the following experiments were performed. First, we performed fate mapping studies using the pan-leukocyte marker CD45, which has two isoforms, CD45.1 and CD45.2; these can be distinguished from each other by monoclonal antibodies (outline in Figure 5A) (35). Starting at 4 weeks of age, CD45.1 Apoe -/- $^{-}$mice were fed a WD for 14 weeks to induce the development of advanced atherosclerotic lesions (baseline group). The aortic arches were then transplanted into WT, $\mathrm{Crr}^{-/-}$, or $\mathrm{Crr}^{-/-}$mice on a CD 45.2 background maintained on a chow diet. We omitted the $\mathrm{C} x 3 \mathrm{CrI}^{-/-}$group, because the previous results in Figures 1 and 2 were essentially the same as in $\mathrm{Ccr}^{-1-}$ mice or in mice treated with the CCR2 inhibitor.

Consistent with Figure 1, the plaques in aortic arches transplanted into $\mathrm{Crr}^{-/-}$mice showed impaired regression compared with those transplanted into WT and $\mathrm{Ccr}^{-/-}$mice (data not shown). There was also a significant reduction in CD $45.1^{+}$donor-derived cells in plaques transplanted into WT and $\mathrm{Crr}^{-/-}$, but not $\mathrm{Ccr} 2^{--}$, mice (Figure 5, B and C). Conversely, there was significant accumulation of $\mathrm{CD} 45.2^{+}$newly recruited recipient-derived cells, compared with baseline, in plaques transplanted into WT and $\mathrm{Ccr} 5^{-1-}$, but not $\mathrm{Crr}^{--}$, mice (Figure 5, B and C). As shown in Figure 5D, $\sim 80 \%$ of the $\mathrm{MR}^{+}$cells in the plaques in the WT recipient group were of CD45.2 (recipient-derived) origin. Overall, the data are consistent with the impaired regression in $\mathrm{Ccr}^{-/-}$mice being due to less recruitment of recipient monocytes, and that the newly recruited monocytes become polarized overwhelmingly to the M2 macrophage state.

Next, we transplanted aortic arches from $\mathrm{Apoe}^{-/-}$mice that were fed the WD for 16 weeks (baseline group) into CD68-GFP transgenic mice, a model recently introduced for tracking CD68 ${ }^{+}$ monocyte-derived cells, including in atherosclerosis (outline in Figure $5 \mathrm{E})(36,37)$. After 5 days, the transplanted arches were excised and enzymatically digested to release cells from the plaques. Using surface marker labeling and flow cytometry, we measured the content of $\mathrm{M} 2$ macrophages by gating on $\mathrm{CD} 45^{+} \mathrm{CD}-$ $11 \mathrm{~b}^{+} \mathrm{F} 4 / 80^{+} \mathrm{MR}^{+}$cells. Notably, we found that $73.9 \% \pm 6.5 \%(n=$ 3) of the M2 macrophages in regressing plaques were CD68-GFP ${ }^{+}$ (representative analysis shown in Figure 5F), indicating that the majority of those macrophages originated from the recipient, and not donor, mice.

To extend these findings, we also isolated $\mathrm{CD} 45^{+} \mathrm{CD}$ $11 \mathrm{~b}^{+} \mathrm{F} 4 / 80^{+}$macrophages from aortic arches from mice in baseline and regression groups subjected to aortic digestion and FACS. Single macrophages were then captured in a Fluidigm $\mathrm{C} 1$ instrument for single-cell RNA sequencing (RNA-seq) analysis. As outlined in Figure 5G, only newly recruited cells from the WT recipient mice will express Apoe mRNA, thus allowing us to use it as a marker for macrophage origin during regression. Compared with baseline, a large proportion of aortic macrophages from regressing plaque samples expressed both Apoe and Cd206 (encoding MR), as well as Apoe and Arg1 mRNAs (Figure 5H). This shows at a single-cell level that M2 macrophages in regressing plaques originate from newly recruited monocytes. Together, these data from three different approaches strongly support that there is recruitment of monocytes in transplant recipient mice and that they are the sources of the M2 macrophages in regressing plaques. 
A

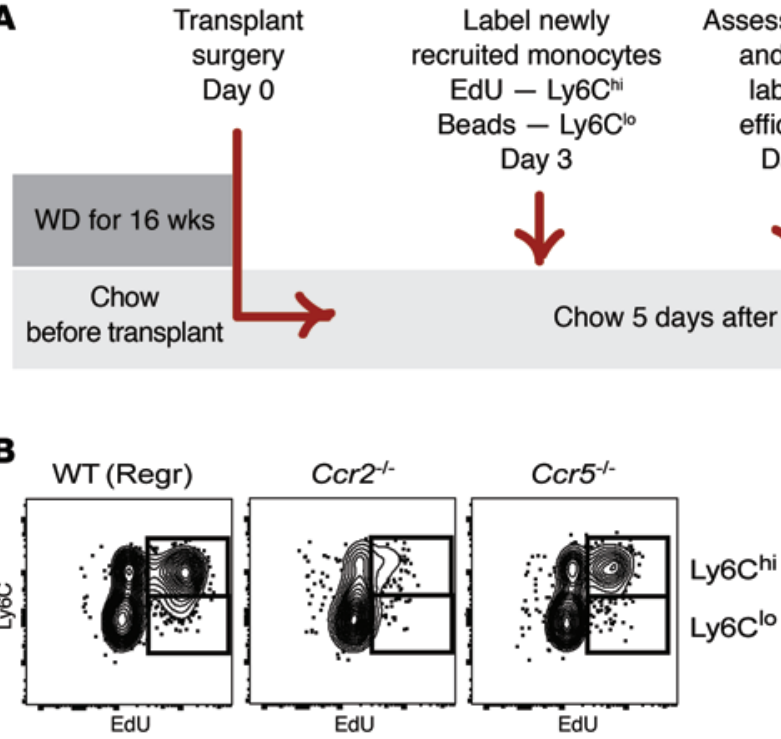

$\mathbf{E}$

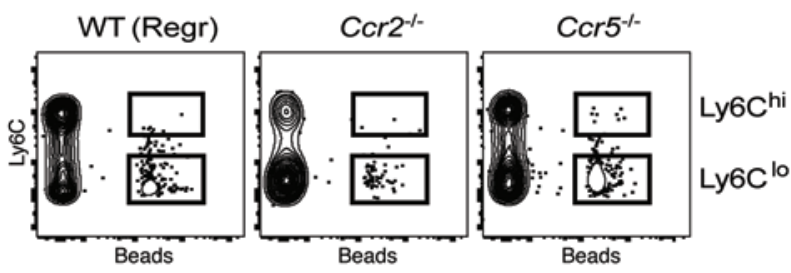

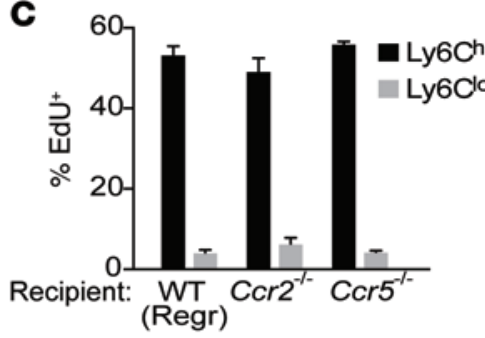

$\mathbf{F}$

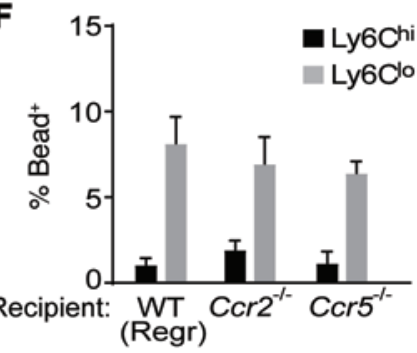

H

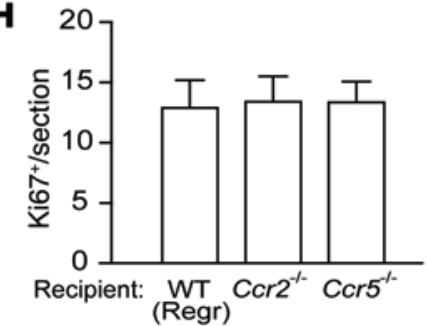

Sacrifice

recipients

Ly6C $\mathrm{C}^{\text {hi recruitment }-\mathrm{EdU}+}$

Ly6C ${ }^{\circ}$ recruitment - Bead $^{+}$

\section{Day 4 Day 5}

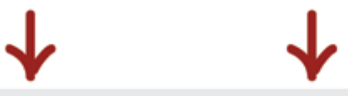

\section{ant}


A

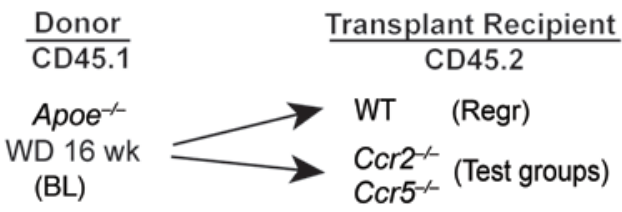

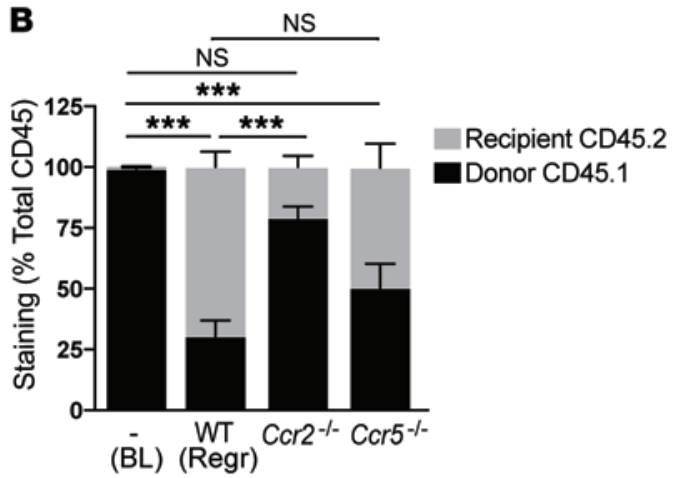
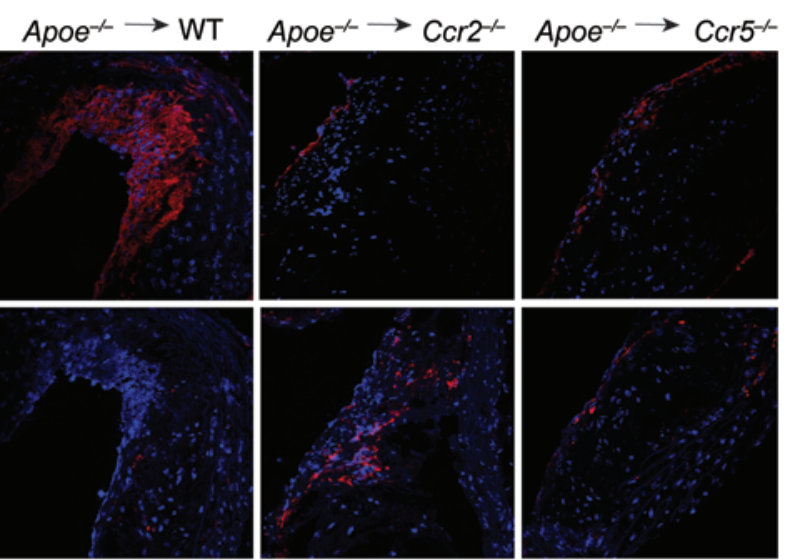

D

Recipient CD45.2 Donor CD45.1
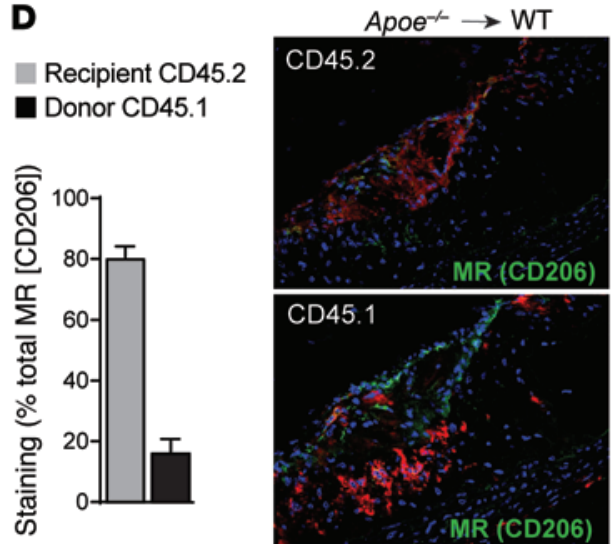

E

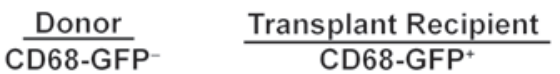

Apoe $^{-/}$

WD 16 wk $\longrightarrow$ WT (Regr)

(BL)
F

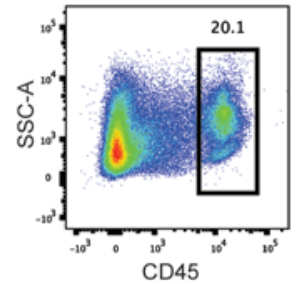

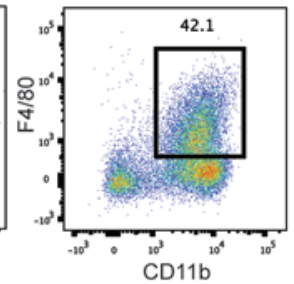

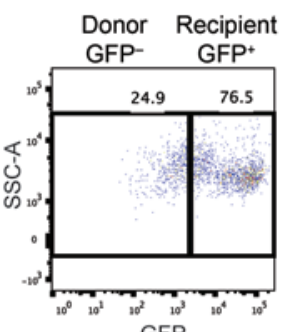

GFP

G

$\frac{\text { Donor }}{\text { Apoe }^{-}} \quad \frac{\text { Transplant Recipient }}{A p o e^{+}}$

Apoe ${ }^{-1}$

WD $16 \mathrm{wk} \longrightarrow$ WT (Regr)

(BL)

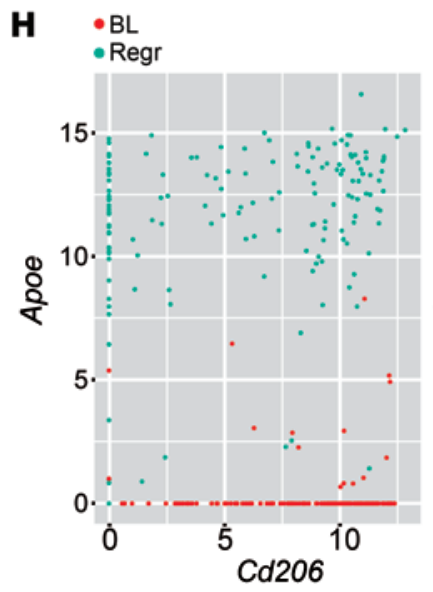

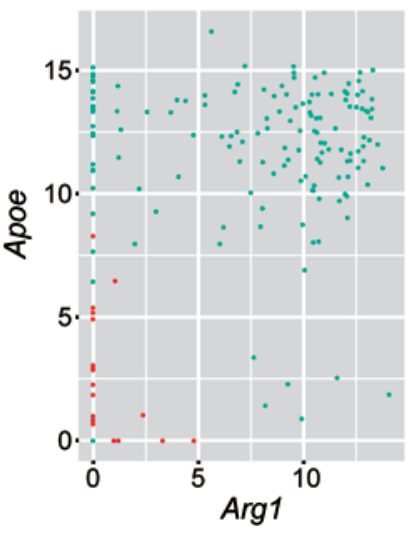

Figure 5. CCR2 deficiency in recipient leukocytes impairs their recruitment to atherosclerotic plaques, where they normally display M2 characteristics. (A) Schematic of CD45.1 (donor) to CD45.2 (recipient) aortic transplantation experiments with (B) quantification of immunohistochemical staining of CD45.1 and CD45.2 in aortic arch plaques from Apoe $^{-/-}$mice on 14-week WD (BL; $n=7$ ) or 5 days after transplant into WT mice (Regr; $n=8$ ), or chemokine receptor-KO recipient mice (C $C r 2^{-1-}$ or $\left.\mathrm{CrF}^{-1-} ; n=8\right)$; ${ }^{* *} P<0.001$ for the indicated comparisons group using 1-way ANOVA with Dunnett's multiple comparisons testing. (C) Representative images of aortic plaques stained for CD45.1 and CD45.2, imaged at $\times 40$ quantification. (D) Quantification of immunohistochemical staining of $\mathrm{MR}^{+}$with CD45.2 or CD45.1 showing $80.72 \% \pm 3.597 \% \mathrm{MR}^{+}$cells originate from recipient $\mathrm{CD} 45.2$ mice $(n=3)$ with representative images at $\times 40$ magnification. (E) Schematic of transplantation experiment using CD68-GFP reporter mice to mark recipient monocytes/macrophages. (F) Representative flow cytometry plots showing that a majority of $\mathrm{CD}^{2} 5^{+} \mathrm{CD} 11 \mathrm{~b}^{+} \mathrm{F} 4 / 80^{+} \mathrm{MR}^{+}$macrophages are GFP+ in aortic arches harvested 5 days after transplantation $(n=3)$. (G) Schematic of transplant experiment for single cell RNA-seq experiments, with $(\mathbf{H})$ scatter plots showing, at a single-cell level, that cells expressing high levels of Apoe (i.e., recipient) are also positive for high levels of $M 2$ macrophage markers $C d 206$ (MR) and Arg1 in the population of $C d 11 b^{+} F 4 / 80^{+}$macrophages isolated from aortic arches 3 days after transplantation. 

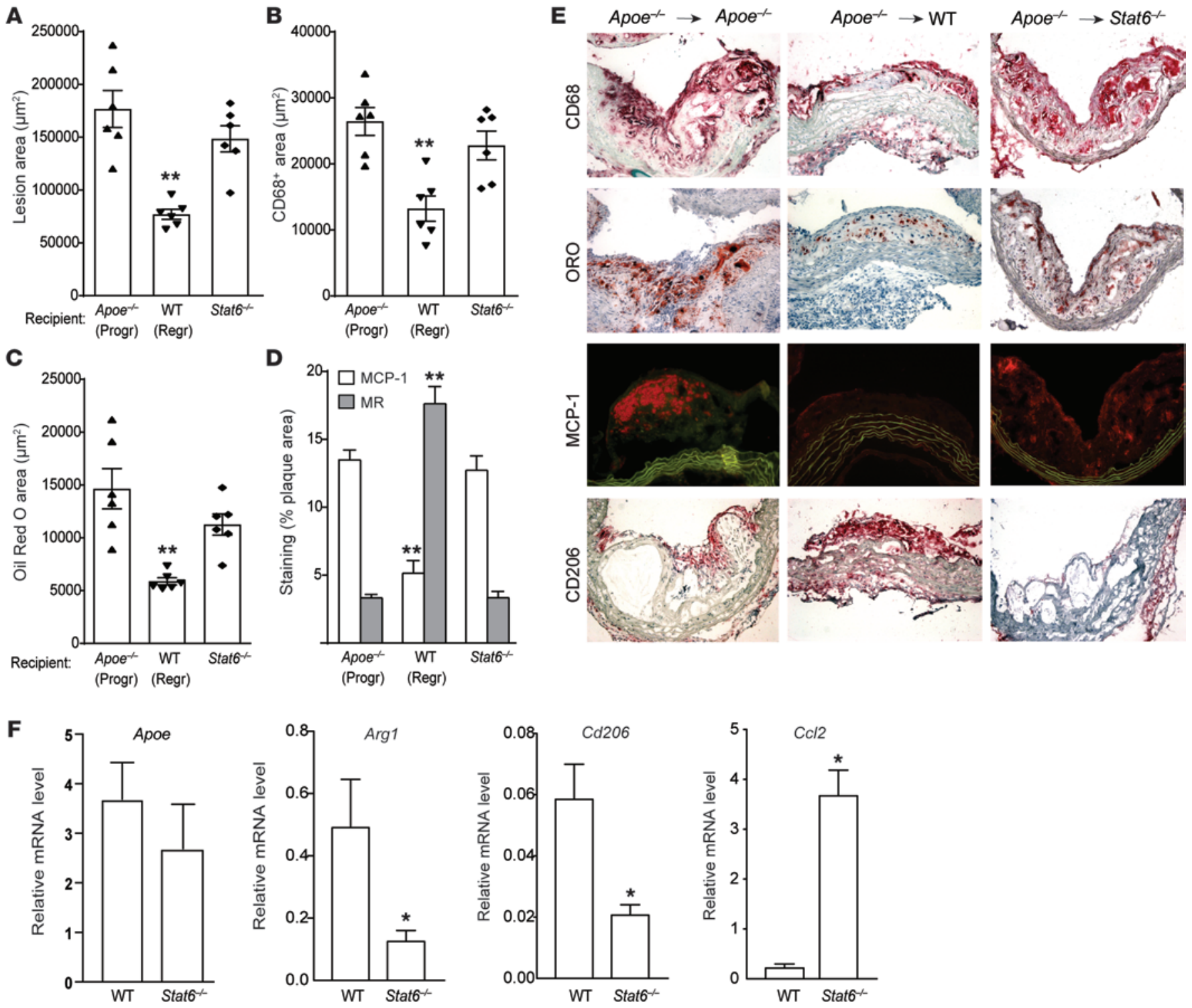

Figure 6. Lack of STAT6 in recipient mice prevents M2 macrophage enrichment of plaques and impairs atherosclerosis regression. Analysis of aortic arch plaques from mice 3 days after transplantation into $A p o e^{-/-}$(Progr; $\left.n=6\right)$, WT (Regr; $n=6$ ), or Stat6 - $^{--}(n=6)$ mice for $(\mathbf{A})$ lesion area, (B) immunohistochemical staining for the macrophage marker $C D 68$, (C) Oil red 0 staining for neutral lipid, and (D) immunohistochemical staining for the M1 macrophage marker MCP-1 and the M2 macrophage marker MR (CD206); ${ }^{* *} P<0.001$ compared with Apoe ${ }^{-/-}$progression group using 1-way ANOVA with Dunnett's multiple comparisons testing. (E) Representative images of aortic plaques stained for CD68, Oil red O (ORO), MCP-1, and CD206, imaged at $\times 40$ magnification. (F) qRT-PCR analysis of mRNA expression of newly recruited monocyte-derived macrophage marker (Apoe), CD206 (M2) (Arg1 and Cd206), and M1 (Cc/2) macrophage markers in CD68 $8^{+}$cells laser captured from the aortic arch plaques 3 days after transplant into WT and Stat6 ${ }^{-1-}$ recipient mice ( $n=4-7$ per group). ${ }^{*} P<0.05$ using unpaired $t$ testing.

M2 macrophage marker enrichment in plaques despite their normolipidemic environment (Figure 6, D and E). Isolation of plaque $\mathrm{CD} 8^{+}$cells by laser capture microdissection confirmed higher mRNA levels of the M1 marker Ccl2 (encoding MCP-1) and lower mRNA levels of the M2 markers Arg1 and Cd206 (encoding MR) in STAT6-deficient recipients compared with WT (Figure 6F). Notably, levels of Apoe mRNA, which, as noted above, is only expressed in newly recruited monocyte-derived macrophages from recipient mice, were not different in plaques transplanted into Stat $^{-/-}$and WT recipient mice, suggesting similar levels of monocyte recruitment (Figure 6F). Thus, these data taken with the other results support a model in which atherosclerosis regression is mediated by newly recruited Ly6 $\mathrm{C}^{\text {hi }}$ monocytes that polarize to M2 macrophages in a STAT6-dependent manner.

\section{Discussion}

Atherosclerosis is a chronic inflammatory disease that develops in the setting of hyperlipidemia, with progression a consequence of the failure to resolve inflammation (38). Using a number of mouse models of atherosclerosis regression, including the aortic arch transplant used in the present study, we have previously shown that aggressive lipid lowering promotes the resolution of plaque 
inflammation, which is characterized by a decreased content of macrophages and an increase in the level of markers of the M2 state $(2,4,5,12)$. We now extend these findings to show that plaque regression and the attendant resolution of inflammation surprisingly require the recruitment of new monocytes, which assume the characteristics of M2 macrophages. Furthermore, contrary to the prevailing paradigm (16), the newly recruited monocytes are drawn from the Ly $6 \mathrm{C}^{\text {hi }}$ circulating subset, generally considered to be "inflammation-prone" precursors of M1 macrophages.

These findings are based on a number of experimental approaches, which include aortic transplantation of atherosclerotic aortae into recipient mice with normolipidemia and deficiencies of various chemokine receptors; monocyte/macrophage trafficking methods; the administration of an inhibitor of CCR2-mediated $\mathrm{Ly} 6 \mathrm{C}^{\text {hi }}$ monocyte recruitment; the differentiation of donor from recipient cells by CD45.1/45.2 fate mapping; determination of CD68-driven GFP expression; and single-cell RNA-seq of plaque macrophages. Together, these data provide a strong foundation for our major conclusion, namely that atherosclerosis regression after lipid lowering is dependent on the recruitment of Ly6 $\mathrm{C}^{\mathrm{hi}}$ monocytes and their polarization toward the M2 phenotype. Furthermore, the data also strongly suggest that Ly $6 \mathrm{C}^{\text {lo }}$ monocyte-derived cells cannot replace the required Ly6C ${ }^{\text {hi }}$ monocytes in promoting plaque regression. While we have reported the enrichment in plaques of the M2 phenotype in a number of mouse models of atherosclerosis regression $(2-4,5,12)$, we recognize that the present results are based on a specific aortic transplantation model. Whether they explain the M2 phenotypic enrichment more generally in other murine models of atherosclerosis regression or apply to the clinical setting will require additional investigation.

The characteristic rapid reversal of hyperlipidemia in mouse atherosclerosis regression models is likely to reduce the continuous stimulation of the plaque inflammatory response by atherogenic lipoproteins, but clearly is not sufficient for the resolution of inflammation. Based on our results, M2 enrichment must also occur, and how the change in lipoprotein environment causes this to happen also remains to be determined. Our finding that it depends on STAT6-dependent signaling in the newly recruited monocytes suggests that local factors in the regressing plaque stimulate this signaling pathway. STAT6 is activated by two key cytokines, IL-4 and IL-13. However, which of these cytokines is the main player, as well as their cellular source(s), in promoting plaque regression is unclear.

A number of potential sources of these cytokines exist, including Th2 lymphocytes, which can accumulate in plaques (39). Indeed, we have shown that adaptive $\mathrm{CD} 4^{+} \mathrm{T}$ cells are required for maintenance of M2 macrophages during chronic helminth infection (40). Another possibility is suggested by the finding that during acute allergic skin inflammation, basophils are a source of the IL-4 that polarizes newly recruited Ly6 $\mathrm{C}^{\text {hi }}$ monocytes to M2 macrophages (28). Furthermore, eosinophils have been identified as a source of IL-4/IL-13 for M2 macrophage polarization in adipose tissue under steady-state conditions and during acute helminth infection, and invariant NKT (iNKT) cells provide IL-4 to promote monocyte differentiation to M2 macrophages in experimental autoimmune encephalomyelitis (EAE) (41). Which of these immune cell subtypes is important for M2 polarization signaling in atherosclerosis regression is being actively investigated by our laboratory.
Though many questions remain, the present results provide insights into the dynamic nature of the inflammatory process and the role of Ly $6 \mathrm{C}^{\text {hi }}$ monocytes in plaques. These cells were previously thought to contribute only to plaque progression and inflammation, but are now shown here to be important in regression and inflammation resolution. One clinically relevant insight raised by our studies is that strategies that promote the accumulation of M2 macrophages in atherosclerotic lesions may be a promising approach toward promoting plaque regression, consistent with recent studies in mice in which treatment with IL-13-or IL-4-based therapy was protective against atherosclerosis progression $(42,43)$.

\section{Methods}

Animals and aortic transplantation. The aortic arch transplantation model has been described previously $(5,6,8,12,17,24)$. Briefly, a donor arch from an atherosclerotic mouse was interpositioned with the abdominal aorta in the recipient mouse, and blood flow was directed through the graft. The donor Apoe $e^{--}$(B6.129P2-Apoe tmiUnc/J) mice, in either a CD45.1 (Apoe - $^{--}$crossed with B6.SJL-Ptprc ${ }^{a} P e p c^{b}$ /BoyJ) or CD45.2 background, were weaned at 4 weeks onto a $21 \%$ fat, $0.3 \%$ cholesterol WD (Dyets Inc.) for 14-16 weeks. Mice were then divided into one group (pre-transplant) for baseline analyses and another group to be donors of aortic arch segments. The recipients were WT (C57BL/6), CD68GFP reporter (C57BL/6-Tg(CD68-EGFP)1Drg/J), Apoe-/, Ccr2 ${ }^{-/-}$ (B6.129S4-Ccr2 $\left.{ }^{\text {tmlffc}} / \mathrm{J}\right), \quad C x 3 \mathrm{cr}^{-/-} \quad\left(\mathrm{B} 6.129 \mathrm{P}-\mathrm{C} x 3 \mathrm{cr}^{\text {tmlLitt }} / \mathrm{J}\right), \quad \mathrm{Ccr} 5^{-/-}$

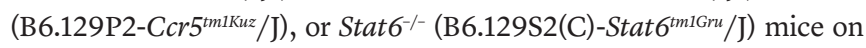
a CD45.2 background (purchased from The Jackson Laboratory). All recipient mice were 20 weeks old and maintained on standard chow diet, and were sacrificed 3 or 5 days after transplantation. In some experiments, mice were administered an inhibitor of CCR2 (CCX417), which was provided by ChemoCentryx. $10 \mathrm{mg} / \mathrm{ml}$ stock of CCX417 was used at a dose of $5 \mathrm{ml} / \mathrm{kg}$ subcutaneously once a day, as instructed by ChemoCentryx, and administered for 48 hours before and 72 hours after the transplantation.

Single-cell RNA-seq cell isolation and flow cytometry analysis. $\mathrm{CD} 45^{+} \mathrm{CD} 11 \mathrm{~b}^{+} \mathrm{F} 4 / 80^{+}$macrophages were isolated using the $\mathrm{BD}$ FACS Aria IIu SORP from pre-transplant baseline and grafted arches from WT recipient mice (regression) that were removed after perfusion of cold PBS and enzymatic digestion as described in ref. 44. Cell surface markers were labeled with the following antibodies: CD45 PerCpCy5.5 (BioLegend, catalog 103132), CD11b AF700 (BioLegend, catalog 101222), and F4/80 PeCy7 (BioLegend, cata$\log 123114)$. Single cells were captured and processed on a Fluidigm C1 instrument in collaboration with Regeneron Pharmaceuticals as described in ref. 45 .

For analysis of cells isolated from grafted arches from CD68-GFP reporter mice, samples were processed as described above, and flow cytometry was conducted on a BD LSRII UV. Cell surface markers were labeled with CD45 PerCpCy5.5 (BioLegend, catalog 103132), CD11b BV650 (BioLegend, catalog 101259), F4/80 PeCy7 (BioLegend, catalog 123114), and MR/CD206 APC (BioLegend, catalog 141712).

Single-cell RNA-seq analysis. Single-cell RNA-seq reads were mapped to the reference mouse genome (GRCm38), including multiple mapped reads, using ArrayStudio. Estimated counts of reads that mapped within each of 24,475 RefSeq genes, taking into account read-mapping uncertainty, were obtained using the RNA-Seq by Expectation Maximization (RSEM) algorithm (46) in ArrayStudio (OmicSoft). We carried out all sub- 
sequent RNA-seq analysis steps in the R statistical computing environment (version 3.2.1; https://www.r-project.org/). We excluded from further analysis all genes for which the count did not exceed zero in at least two samples. For the remaining 16,294 genes, we rounded the expression counts to the nearest integer and then adjusted the count values on a per-sample basis using DESeq2 (http://bioconductor.org/packages/ release/bioc/html/DESeq2.html) normalization, with size factors based on the sample-wise geometric means of the counts for genes with nonzero counts in each sample. We then quantile-normalized (47) the count data in order to adjust for batch effects. For the global unsupervised two-dimensional visualization of the RNA-seq data, we transformed the normalized count matrix by adding one to each entry and then $\log _{2}$ transforming. We used multidimensional scaling (MDS) (48) with $n=2$ dimensions (including only genes for which the normalized count was 50 or greater in at least one sample) in order to obtain an unsupervised two-dimensional visualization of the $\log _{2}$-transformed gene expression data on a sample-specific basis. We further eliminated any sample from downstream analysis if its sum of $\log _{2}$ counts was not at least 1,200. The above sample assignment and filtering steps yielded 536 macrophage samples. Next, we filtered samples based on library size, numbers of expressed genes, and mitochondrial gene counts, as recommended for single-cell RNA-seq analysis (49). For library size, we eliminated samples with extremely low sum $\log _{2}$ counts across all genes (with the cutoff defined as the sample-wise median of the sum $\log _{2}$ count minus three times the sample-wise median absolute deviation) (49). For numbers of expressed genes, we eliminated samples with an extremely low number of genes with nonzero counts (with the cutoff defined as the sample-wise median minus three times the sample-wise median absolute deviation). Finally, we eliminated any sample in which the percentage of counts that were in mitochondrial genes was anomalously high (with the threshold defined as the sample-wise median plus three times the sample-wise median absolute deviation). After this filtering, 452 macrophage samples remained. We tested genes for differential expression using the edgeR software package (50), using the general linear model (GLM) approach including normalization, which enabled us to compare progression to baseline, regression to baseline, and regression to progression sample groups. We used a minimum absolute $\log _{2}$ ratio of 1.0 for filtering for differential expression, in addition to thresholding the $P$ value for differential expression to limit the estimated false discovery rate (for each of the sample group comparisons) to $5 \%$ based on the Benjamini-Hochberg method (51). Data submitted to NCBI GEO; accession number, GSE97941.

Lipid and lipoprotein analyses. Plasma total cholesterol levels were determined by enzymatic assays (Wako Life Sciences). Plasma HDL cholesterol was determined by precipitating non-HDL cholesterol (Wako Diagnostic) and then assaying the remaining cholesterol with Infinity Total Cholesterol Reagent.

Immunohistochemistry and plaque assessment. The pre-transplant baseline and grafted arches were removed after perfusion of cold PBS, embedded in OCT, and frozen. Serial sections (6 $\mu \mathrm{m}$ thick) were cut and stained for CD68 (AbD Serotec, catalog MCA1957) to detect monocyte-derived cells as previously described (22). The same immunostaining protocol with different primary antibodies was used in order to detect CD206, widely known as mannose receptor (MR) (AbD Serotec, catalog MCA2235), ARG1 (Santa Cruz Biotechnology Inc., catalog sc-20150), MCP-1 (BioLegend, catalog 505908), CD45.1 (SouthernBiotech, catalog 1795-08), CD45.2 (SouthernBiotech, catalog 1800-08), Ki67 (Abcam, catalog Ab 16667), and cleaved caspase 3 (Cell Signaling Technology, catalog 9661S). ImagePro Plus 7.0 software (Media Cybernetics) was used to determine $\mathrm{CD}^{+} 8^{+}, \mathrm{MR}^{+}, \mathrm{ARG}^{+}$, and $\mathrm{MCP}-1^{+}$areas. Fiji (52) was used to determine CD $45.1^{+}$and CD $45.2^{+}$areas as well as to identify Ki67 ${ }^{+}$ and cleaved caspase $3^{+}$cells in the plaques. $\mathrm{CD} 68^{+}$cells were selected from atherosclerotic plaques by laser capture microdissection as previously described (24). RT-PCR was performed using a 7300 Real-Time PCR System (Applied Biosystems) and primers listed in ref. 12.

Monocyte subset labeling. Mice were injected intraperitoneally with 1 $\mathrm{mg} / \mathrm{mouse}$ EdU (Life Technologies, catalog E10187) to label the Ly6 $\mathrm{C}^{\text {hi }}$ subset of monocytes. EdU incorporation into circulating monocytes was detecting using the Click-iT Plus EdU Pacific Blue Flow Cytometry Assay Kit (Life Technologies, catalog C10636) and in plaque tissue using the Click-iT Plus EdU Alexa Fluor 647 Imaging Kit (Life Technologies, catalog C10640). Mice were injected retro-orbitally with green fluorescent latex beads to label $\mathrm{Ly} 6 \mathrm{C}^{\mathrm{lo}}$ monocytes. Their incorporation into circulating monocytes was detected and number of beads/ section assessed as described in refs. 8, 17, 19. Circulating monocytes were detected by flow cytometry using surface markers CD45 PE/Cy7 (BioLegend, catalog 103114), CD115 PE (BioLegend, catalog 135506) or APC (BioLegend, catalog 135510), and Ly6C APC (BioLegend, catalog 108412) or PerCP Cy5.5 (BD Bioscience, catalog 560525).

Statistics. Data are expressed as mean \pm SEM. Data were typically analyzed by 1-way ANOVA (GraphPad Prism 7), with Dunnett's multiple comparisons testing when there were 3 or more groups. Data involving comparisons between 2 groups were analyzed using unpaired $t$ testing (GraphPad Prism 7). $P \leq 0.05$ was considered significant.

Study approval. These studies were performed in strict accordance with the recommendations in the NIH Guide for the Care and Use of Laboratory Animals (National Academies Press, 2011). The Institutional Animal Care and Use Committee of the New York University School of Medicine reviewed and approved the protocol.

\section{Author contributions}

KR, YV, KJM, PL, and EAF designed experiments. KR, YV, and EAF wrote the draft of the manuscript, followed by editing by KJM and PL. KR, YV, NRV, NMG, and AW performed experiments. JL performed transplant surgeries on all mouse groups. VG and JG coordinated and performed the single-cell RNA-seq experiments. SAR analyzed single-cell RNA-seq data.

\section{Acknowledgments}

This work was supported by NIH grant HL084312 (EAF, PL, and KJM); NIH training grant T32HL098129 (YV); and Comisionado para Universidades e Investigación (CUR), DIUE, Generalitat de Catalunya and Fulbright program (NRV). KR was supported by NIH training grants T32GM007308 and T32AI100853, and NIH fellowship F30HL131183. PL was supported by grants from the NIH (AI093811 and AI094166), and NMG was supported by NIH fellowship F32AI102502 and training grant T32AI007180. SAR was supported by NSF grants 1557605-DMS and 1553728-DBI, Medical Research Foundation of Oregon New Investigator Grant, PhRMA Foundation Informatics Grant, and Oregon State University Division of Health Sciences Interdisciplinary Research Grant. We also acknowledge Yi Wei, Qi Su, and Yurong Xin of Regeneron Pharmaceuticals for their work on the single-cell RNA-seq experiments, as well as Yaritzy Astudillo, Yoscar Ogando, and Sheela George of the Fisher laboratory for their technical contributions. 
Address correspondence to: Edward A. Fisher, Department of Medicine, Division of Cardiology/Marc and Ruti Bell Program in Vascular Biology, New York University School of Medicine, Smilow, Room 704, 522 First Avenue, New York, New York 10016, USA. Phone: 212.263.6631; Email: edward.fisher@ nyumc.org. Or to: P'ng Loke, Department of Microbiology, New York University School of Medicine, Alexandria Center for Life Sciences, AW Room 314, 430 East 29th Street, New York, New York 10016, USA. Phone: 646.501.4649; Email: png.loke@ nyumc.org.
1. Williams KJ, Feig JE, Fisher EA. Rapid regression of atherosclerosis: insights from the clinical and experimental literature. Nat Clin Pract Cardiovasc Med. 2008;5(2):91-102.

2. Feig JE, et al. Reversal of hyperlipidemia with a genetic switch favorably affects the content and inflammatory state of macrophages in atherosclerotic plaques. Circulation. 2011;123(9):989-998.

3. Hewing B, Parathath S, Mai CK, Fiel MI, Guo L, Fisher EA. Rapid regression of atherosclerosis with MTP inhibitor treatment. Atherosclerosis. 2013;227(1):125-129.

4. Rayner KJ, et al. Antagonism of miR-33 in mice promotes reverse cholesterol transport and regression of atherosclerosis. J Clin Invest. 2011;121(7):2921-2931.

5. Feig JE, et al. Regression of atherosclerosis is characterized by broad changes in the plaque macrophage transcriptome. PLoS One. 2012;7(6):e39790.

6. Reis ED, et al. Dramatic remodeling of advanced atherosclerotic plaques of the apolipoprotein E-deficient mouse in a novel transplantation model. J Vasc Surg. 2001;34(3):541-547.

7. Chereshnev I, et al. Mouse model of heterotopic aortic arch transplantation. JSurg Res. 2003;111(2):171-176.

8. Feig JE, et al. LXR promotes the maximal egress of monocyte-derived cells from mouse aortic plaques during atherosclerosis regression. J Clin Invest. 2010;120(12):4415-4424.

9. Moore KJ, Sheedy FJ, Fisher EA. Macrophages in atherosclerosis: a dynamic balance. Nat Rev Immunol. 2013;13(10):709-721.

10. Gordon S, Martinez FO. Alternative activation of macrophages: mechanism and functions. Immunity. 2010;32(5):593-604.

11. Sica A, Mantovani A. Macrophage plasticity and polarization: in vivo veritas. J Clin Invest. 2012;122(3):787-795.

12. Feig JE, et al. HDL promotes rapid atherosclerosis regression in mice and alters inflammatory properties of plaque monocyte-derived cells. Proc Natl Acad Sci USA . 2011;108(17):7166-7171.

13. Parathath S, et al. Diabetes adversely affects macrophages during atherosclerotic plaque regression in mice. Diabetes. 2011;60(6):1759-1769.

14. Khallou-Laschet J, et al. Macrophage plasticity in experimental atherosclerosis. PLOS ONE. 2010;5(1):e8852.

15. Jenkins SJ, et al. Local macrophage proliferation, rather than recruitment from the blood, is a signature of TH2 inflammation. Science. 2011;332(6035):1284-1288.

16. Geissmann F, Manz MG, Jung S, Sieweke MH, Merad M, Ley K. Development of monocytes, macrophages, and dendritic cells. Science. 2010;327(5966):656-661.

17. Llodrá J, Angeli V, Liu J, Trogan E, Fisher EA, Randolph GJ. Emigration of monocyte-derived cells from atherosclerotic lesions characterizes regressive, but not progressive, plaques. Proc Natl Acad Sci U S A. 2004;101(32):11779-11784.

18. Swirski FK, et al. Ly-6Chi monocytes dominate hypercholesterolemia-associated monocytosis and give rise to macrophages in atheromata. J Clin Invest. 2007;117(1):195-205.

19. Tacke F, et al. Monocyte subsets differentially employ CCR2, CCR5, and CX3CR1 to accumulate within atherosclerotic plaques. JClin Invest. 2007;117(1):185-194.

20. Nahrendorf $\mathrm{M}$, et al. The healing myocardium sequentially mobilizes two monocyte subsets with divergent and complementary functions. JExp Med. 2007;204(12):3037-3047.

21. Auffray C, Sieweke MH, Geissmann F. Blood monocytes: development, heterogeneity, and relationship with dendritic cells. Annu Rev Immunol. 2009;27:669-692.

22. Combadière $\mathrm{C}$, et al. Combined inhibition of CCL2, CX3CR1, and CCR5 abrogates Ly6C(hi) and Ly6C(lo) monocytosis and almost abolishes atherosclerosis in hypercholesterolemic mice. Circulation. 2008;117(13):1649-1657.

23. Van Dyken SJ, Locksley RM. Interleukin-4- and interleukin-13-mediated alternatively activated macrophages: roles in homeostasis and disease. Annu Rev Immunol. 2013;31:317-343.

24. Trogan E, et al. Gene expression changes in foam cells and the role of chemokine receptor CCR7 during atherosclerosis regression in ApoE-deficient mice. Proc Natl Acad Sci USA. 2006;103(10):3781-3786.

25. Boring L, Gosling J, Cleary M, Charo IF. Decreased lesion formation in CCR2-/- mice reveals a role for chemokines in the initiation of atherosclerosis. Nature. 1998;394(6696):894-897.

26. Saederup N, Chan L, Lira SA, Charo IF. Fractalkine deficiency markedly reduces macrophage accumulation and atherosclerotic lesion formation in CCR2-/- mice: evidence for independent chemokine functions in atherogenesis. Circulation. 2008;117(13):1642-1648.

27. Tsou CL, et al. Critical roles for CCR2 and MCP-3 in monocyte mobilization from bone marrow and recruitment to inflammatory sites. JClin Invest. 2007;117(4):902-909.

28. Egawa M, et al. Inflammatory monocytes recruited to allergic skin acquire an anti-inflammatory M2 phenotype via basophil-derived interleukin-4. Immunity. 2013;38(3):570-580.

29. Girgis NM, Gundra UM, Ward LN, Cabrera M, Frevert U, Loke P. Ly6C(high) monocytes become alternatively activated macrophages in schistosome granulomas with help from CD4+ cells. PLoS Pathog. 2014;10(6):e1004080.

30. Gundra UM, et al. Alternatively activated macrophages derived from monocytes and tissue macrophages are phenotypically and functionally distinct. Blood. 2014;123(20):e110-e122.
31. Hilgendorf I, et al. Ly-6Chigh monocytes depend on Nr4a1 to balance both inflammatory and reparative phases in the infarcted myocardium. Circ Res. 2014;114(10):1611-1622.

32. Nagareddy PR, et al. Hyperglycemia promotes myelopoiesis and impairs the resolution of atherosclerosis. Cell Metab. 2013;17(5):695-708.

33. Zhu SN, Chen M, Jongstra-Bilen J, Cybulsky MI. GM-CSF regulates intimal cell proliferation in nascent atherosclerotic lesions. J Exp Med. 2009;206(10):2141-2149.

34. Tagliani E, Shi C, Nancy P, Tay CS, Pamer EG, Erlebacher A. Coordinate regulation of tissue macrophage and dendritic cell population dynamics by CSF-1. JExp Med. 2011;208(9):1901-1916.

35. Xu H, Exner BG, Chilton PM, Schanie C, Ildstad ST. CD 45 congenic bone marrow transplantation: evidence for T cell-mediated immunity. Stem Cells. 2004;22(6):1039-1048.

36. Iqbal AJ, et al. Human CD68 promoter GFP transgenic mice allow analysis of monocyte to macrophage differentiation in vivo. Blood. 2014;124(15):e33-e44.

37. McNeill E, et al. Tracking monocyte recruitment and macrophage accumulation in atherosclerotic plaque progression using a novel hCD68GFP/ ApoE-/- reporter mouse - brief report. Arterioscler Thromb Vasc Biol. 2017;37(2):258-263.

38. Tabas I, Glass CK. Anti-inflammatory therapy in chronic disease: challenges and opportunities. Science. 2013;339(6116):166-172.

39. Hansson GK. Immune mechanisms in atherosclerosis. Arterioscler Thromb Vasc Biol. 2001;21(12):1876-1890.

40. Loke $P$, et al. Alternative activation is an innate response to injury that requires $\mathrm{CD} 4+\mathrm{T}$ cells to be sustained during chronic infection. JImmunol. 2007;179(6):3926-3936.

41. Denney L, Kok WL, Cole SL, Sanderson S, McMichael AJ, Ho LP. Activation of invariant NKT cells in early phase of experimental autoimmune encephalomyelitis results in differentiation of Ly6Chi inflammatory monocyte to M2 macrophages and improved outcome. JImmunol. 2012;189(2):551-557.

42. Cardilo-Reis L, et al. Interleukin-13 protects from atherosclerosis and modulates plaque composition by skewing the macrophage phenotype. EMBO Mol Med. 2012;4(10):1072-1086.

43. Wolfs IM, et al. Reprogramming macrophages to an anti-inflammatory phenotype by helminth antigens reduces murine atherosclerosis. FASEB J. 2014;28(1):288-299.

44. Tang J, et al. Inhibiting macrophage proliferation suppresses atherosclerotic plaque inflammation. Sci Adv. 2015;1(3):e1400223.

45. Xin Y, et al. Use of the Fluidigm C1 platform for RNA sequencing of single mouse pancreatic islet cells. Proc Natl Acad Sci U S A. 
2016;113(12):3293-3298.

46. Li B, Ruotti V, Stewart RM, Thomson JA, Dewey CN. RNA-Seq gene expression estimation with read mapping uncertainty. Bioinformatics. 2010;26(4):493-500.

47. Bolstad BM, Irizarry RA, Astrand M, Speed TP. A comparison of normalization methods for high density oligonucleotide array data based on variance and bias. Bioinformatics.
2003;19(2):185-193.

48. Borg I, Groenen PJ. Modern Multidimensional Scaling: Theories and Applications. New York, NY, USA: Springer-Verlag New York; 2005.

49. Lun A, McCarthy D, Marioni J. A step-by-step workflow for low-level analysis of single-cell RNA-seq data. F100ORes. 2016;5:2122.

50. Robinson MD, McCarthy DJ, Smyth GK. edgeR: a Bioconductor package for differential expression analysis of digital gene expression data. Bioinformatics. 2010;26(1):139-140.

51. Benjamini Y, Hochberg Y. Controlling the false discovery rate: a practical and powerful approach to multiple testing. J R Stat Soc Series B Stat Methodol.1995;57(1):289-300.

52. Schindelin J, et al. Fiji: an open-source platform for biological-image analysis. Nat Methods. 2012;9(7):676-682. 